\title{
Neurosurgery as a window to the human mind: free will and the sense of self
}

\author{
Itzhak Fried $^{1}$
}

Received: 28 January 2021 / Accepted: 31 January 2021 / Published online: 5 April 2021

(C) The Author(s), under exclusive licence to Springer-Verlag GmbH, AT part of Springer Nature 2021

As neurosurgeons, we are relentlessly occupied with brain function. Even when busy with the most intricate technical and anatomical details of surgery or perioperative care, the dictum of "First do no harm" is on our mind, translated into a continuous concern for the neurological status of the patient following our surgical intervention or lack thereof. As such, neurosurgeons have a clear map of functional anatomy derived from clinical neurology and neuroscience. Perhaps an oversimplification of such a map is the term "eloquent cortex", a simple bimodal roadmap for the neurosurgeon indicating brain zones where surgery is at risk of producing a noticeable, measurable neurological deficit. These zones included the primary sensory and motor areas and the language and speech areas. The limitations of such approach are evident when one asks: what then is non-eloquent cortex and how should it be treated? $[2,5]$.

In this issue of Acta Neurochirurgica, Schaller et al. [7] ask neurosurgeons to consider a question posed by their patients: "Will I be the same person after surgery?". They proceed to discuss the mapping of the sense of self in neurosurgical patients, a faculty that has not been previously considered in the neurosurgical map of "eloquent cortex". Schaller et al. propose tests to monitor the patient's sense of self, some of which are reasonably practical, such as heartbeat's evoked potentials. Future neurosurgeons may be expected to preserve not only traditional neurological function but also the complex neuropsychological makeup of their patients, including faculties such as the bodily and cognitive sense of self.

However, neurosurgeons have not been merely consumers of neuroscience translated into surgical strategy. There is a strong tradition of insights provided by neurosurgeons that played a major role in shaping our understanding of brain

Itzhak Fried

IFried@mednet.ucla.edu

1 Department of Neurosurgery, University of California Los Angeles, Los Angeles, CA 90095, USA functions. Neurosurgeons have the closest proximity to the human brain and are, therefore, in a privileged position to ask and seek answers to some of the most difficult questions in neuroscience. A single neurosurgical procedure of resection of medial temporal lobe structures on both sides of the brain, performed by William Beecher Scvoville on patient H.M., has had an enormous impact on the study of memory [8]. This was possible only because neurosurgeons were able to document and disseminate their experiences for the scientific and medical community. Thus, neurosurgical direct access to the human brain coupled with direct communication with patients who can declare their thoughts, wishes, and memories can be a source of scientific knowledge inaccessible by any other means. This line of neurosurgical inquiry was practiced by Wilder Penfield with spectacular insights into motor, sensory, language, and memory mechanisms. In recent years there is a growing number of neurosurgeons opening our discipline to neuroscience [1], and there is a substantial increase in public funding directed at such efforts.

In this issue of Acta Neurochirurgica, Sjöberg [10] discusses the supplementary motor area (SMA), largely based on surgical experience with resections in the SMA region, and places it in a cognitive-philosophical perspective pertaining to human free will. The SMA has been a somewhat enigmatic region of the human brain. The so called "SMA syndrome" following resections in this region has often puzzled the postoperative examiner encountering a seemingly hemiplegic patient only to discover later that the deficit is more in the initiation of movement than in actual motoric strength and that these patients recover in few days or weeks. It was indeed a set of neurosurgical observations while using electrical stimulation mapping of this region in epilepsy patients implanted with intracranial electrodes that led to the discovery of somatotopic organization of SMA as well as to the evocation of an urge or will for movement [3]. Sjöberg discusses self-reports by patients who underwent SMA resections for tumor pathology "denying feeling a lack 
of a sense of volition as a reason for their difficulties" [11]. While one may question the validity of such selfreport in patients with "SMA syndrome" who may likely be impaired with initiation of expression, Sjöberg also reports on deficits in several paradigms of executive function administered to these patients [9]. He then combines these observations with critical examination of several studies addressing the role of SMA and preconscious neuronal signatures of volition, most notably Libet's paradigm and subsequent studies [6]. He concludes by arguing against the role of SMA in the sensing of free will and for a role of SMA in the chain of executive control leading to action.

One might argue against extension of such neurosurgical observations to the philosophical questions of free will or lack thereof and to potential implications to legal issues of personal responsibility for one's action. The SMA is only one region in the complex network of human decision that involves much more complexity than a simple movement. Free will involves not only volition to act but also a sense of agency, i.e., the owning of one's acts, a property impaired in conditions such as the alien hand syndrome [4]. At the same time, Sjöberg's critical review as well as Schaller's [7] perspectives underscore the opportunity uniquely available for neurosurgeons, and in fact their obligation, to observe, study, and report on operations of the human mind as they are revealed through their practice. Operations under local anesthesia, placement of intracranial electrodes for monitoring, and the increasing use of deep brain stimulation implants and brain-machine interfaces, all provide extraordinary opportunity for observations, behavioral tests, electrical stimulation, and recordings down to the level of single neurons in the human brain. Neurosurgeons should be vigorous participants and an active voice in the pursuit of the machinery of the human mind.

\section{References}

1. Abbott A (2009) Neuroscience: Opening up brain surgery. Nature 461:866-868

2. Fried I (1993) The myth of eloquent cortex, or what is non-eloquent cortex? J Neurosurg 78(6):1009-1010

3. Fried I et al (1991) Functional organization of human supplementary motor cortex studied by electrical stimulation. J Neurosci 11(11):3656-3666

4. Hassan A, Josephs KA (2016) Alien Hand Syndrome. Curr Neurol Neurosci Rep 16(8):73

5. Kahn E, Lane M, Sagher O (2017) Eloquent: history of a word's adoption into the neurosurgical lexicon. J Neurosurg 127(6):1461

6. Libet $\mathrm{B}$ et al (1983) Time of conscious intention to act in relation to onset of cerebral activity (readiness-potential). The unconscious initiation of a freely voluntary act. Brain 106(Pt 3):623-642

7. Schaller K, Iannotti GR, Orepic P et al (2021) The perspectives of mapping and monitoring of the sense of self in neurosurgical patients. Acta Neurochir. https://doi.org/10.1007/s00701-021-047783

8. Scoville W, Milner B (2009) Bilateral hippocampal lesions loss of recent memory after

9. Sjöberg RL et al (2019) The supplementary motor area syndrome and cognitive control. Neuropsychologia 129:141-145

10. Sjoeberg R (2021) Free will and neurosurgical resections of the supplementary motor area: A critical review. Acta Neurochir. https://doi.org/10.1007/s00701-021-04748-9

11. Stålnacke $\mathrm{M}$ et al (2020) Phenomenology of glioma resection in the dorsal medial frontal cortex. Acta Neurol Scand 142(3):216-220

Publisher's note Springer Nature remains neutral with regard to jurisdictional claims in published maps and institutional affiliations. 